MATHEMATICS OF COMPUTATION

Volume 70, Number 236, Pages 1481-1501

S $0025-5718(00) 01277-1$

Article electronically published on July 11, 2000

\title{
CONVERGENCE OF A SPLITTING METHOD OF HIGH ORDER FOR REACTION-DIFFUSION SYSTEMS
}

\author{
STÉPHANE DESCOMBES
}

\begin{abstract}
In this article, we prove the convergence of a splitting scheme of high order for a reaction-diffusion system of the form $u_{t}-M \Delta u+F(u)=$ 0 where $M$ is an $m \times m$ matrix whose spectrum is included in $\{\mathfrak{R} z>0\}$. This scheme is obtained by applying a Richardson extrapolation to a Strang formula.
\end{abstract}

\section{INTRODUCTION}

Let $N, m$ be two integers. We consider the reaction-diffusion system:

$$
\begin{cases}\frac{\partial u}{\partial t}-M \Delta u+F(u)=0, & x \in \mathbb{R}^{N}, t>0, \\ u(0, x)=u_{0}(x), & x \in \mathbb{R}^{N} .\end{cases}
$$

We suppose that $u$ belongs to $\mathbb{R}^{m}$, that $F$ is a $C^{5}$ function from $\mathbb{R}^{m}$ to itself satisfying

$$
F(0)=0
$$

and that $M$ is an $m \times m$ matrix whose spectrum is included in $\{\mathfrak{R} z>0\}$. Let $\mathbf{L}$ be the space $L^{2}\left(\mathbb{R}^{N}\right)^{m} \cap L^{\infty}\left(\mathbb{R}^{N}\right)^{m}$. We assume that the initial condition $u_{0}$ belongs to $\mathbf{L}$ and that (1.1) has a unique solution belonging to $C([0, \tau], \mathbf{L})$ for all $\tau>0$. We will denote $u(t,$.$) by T^{t} u_{0}$, that is to say $T^{t}$ is the flow associated to (1.1). For example, systems of type (1.1) cover the case of reaction-diffusion systems with symmetric positive definite matrix and the case of complex Ginzburg-Landau equations. We are going to prove the stability and convergence of a splitting method for (1.1). This method is based on the classical decoupling of the diffusion and ODE parts of (1.1), leading to a Strang's formula, which we extrapolate. More precisely, given $v_{0}$ and $w_{0}$ in $\mathbf{L}$, we introduce the following equations:

$$
\begin{cases}\frac{\partial v}{\partial t}-M \Delta v=0, & x \in \mathbb{R}^{N}, t>0, \\ v(0, x)=v_{0}(x), \quad x \in \mathbb{R}^{N}\end{cases}
$$

Received by the editor November 10, 1998 and, in revised form, November 29, 1999. 2000 Mathematics Subject Classification. Primary 65M12, 65B05, 65J15.

Key words and phrases. Splitting, reaction-diffusion systems.

(C)2000 American Mathematical Society 
and

$$
\begin{cases}\frac{\partial w}{\partial t}+F(w)=0, & x \in \mathbb{R}^{N}, t>0, \\ w(0, x)=w_{0}(x), & x \in \mathbb{R}^{N} .\end{cases}
$$

Let $X^{t} v_{0}$ and $Y^{t} w_{0}$ be the respective solutions of (1.3) and (1.4). The motivation for the splitting method that we consider is essentially numerical; the numerical approximation of the solution of a heat type equation and the numerical approximation of a scalar ordinary differential equation are easy, but a numerical integration involving the two operators together is bothersome for the following reasons: if we opt for an explicit scheme, the time step $\Delta t$ is limited by $O\left(\Delta x^{2}\right)$; if we choose an implicit scheme, we have to solve a large system of nonlinear equations which requires the updating of the linearized operator at each time step, a computationally expensive operation.

The Strang approximation formula ([14], [15]) is defined by

$$
Z^{t} u=X^{t / 2} Y^{t} X^{t / 2} u
$$

and is of order two, at least formally. For example, numerical results in the case of complex Ginzburg-Landau equations with periodic boundary conditions are presented in Goldman and Sirovich 7 . Unfortunately, Q. Sheng has proved in 12 that it is generally impossible to generate automatically stable schemes of order higher than two. More precisely, a linear combination with positive coefficients of products of exponentials of the form $\exp (\gamma A t), \exp (\delta B t)$ when $\gamma$ and $\delta$ are positive, and $A$ and $B$ are dissipative is at most an approximation of order two to $\exp (t(A+B))$. As in Goldman and Kaper [8, we propose a scheme of order greater than 2. This scheme is obtained by applying a Richardson's extrapolation to $Z^{t}$ and is given by

$$
W^{t} u=\frac{4}{3} Z^{t / 2} Z^{t / 2} u-\frac{1}{3} Z^{t} u .
$$

It is formally of order 4 [5] and the implementation of this scheme in the case of complex Ginzburg-Landau equations with periodic boundary conditions is presented in Descombes and Schatzman 11. The comparison between error and CPU time favors this scheme over Strang's classical scheme (1.5). Thus the purpose of this paper is to obtain the stability and convergence of this scheme. But the stability of higher order methods requires a much more refined analysis than for (1.5), which we undertake here.

Denote $H$ the Hilbert space $L^{2}\left(\mathbb{R}^{N}\right)^{m}$. We need a contraction property of the operator $X^{t}$ in $H$, which is not true if we use the canonical Euclidean scalar product. Therefore, we equip the space $\mathbb{R}^{m}$ with a noncanonical scalar product. Since $M$ is a matrix whose spectrum is included in $\{\mathfrak{R} z>0\}$, we define the following matrix:

$$
S=\int_{0}^{+\infty} e^{-s M^{*}} e^{-s M} d s .
$$

Denoting by 1 the identity of any algebra of operators, it can be easily checked that $S$ is a symmetric positive definite matrix satisfying

$$
S M+M^{*} S=-\int_{0}^{+\infty} \frac{d}{d s}\left(e^{-s M^{*}} e^{-s M}\right)=1 .
$$


We denote (.|.) the Euclidean scalar product in $\mathbb{R}^{m}$; for any vector $\omega \in \mathbb{R}^{m}, \omega \neq 0$, (1.8) implies

$$
(S M \omega \mid \omega)=\left(\omega \mid M^{*} S \omega\right)=(\omega \mid \omega)-(S M \omega \mid \omega),
$$

and therefore

$$
(S M \omega \mid \omega)=\frac{1}{2}(\omega \mid \omega)>0 .
$$

Hence there exists a constant $\beta>0$ such that for any vector $\omega \in \mathbb{R}^{m}$,

$$
(S M \omega \mid \omega) \geq \beta(S \omega \mid \omega) .
$$

From now on, the scalar product of two vectors $\omega, \eta$ in $\mathbb{R}^{m}$ will be $\omega \cdot \eta=(S \omega \mid \eta)$ and the corresponding vector norm is denoted || . The space $H$ is equipped with the scalar product defined for all $v$ and $w$ in $H$ by

$$
(v \mid w)_{H}=\int_{\mathbb{R}^{N}} v \cdot w d x
$$

the associated norm is denoted $|\cdot|_{H}$ and the operator norm $|\cdot|_{\mathcal{L}(H)}$. For all $v$ in $H$ and $t>0$ the operator $X^{t}$ satisfies

$$
\left|X^{t} v\right|_{H} \leq|v|_{H} .
$$

Let us introduce some other functional spaces: the space $L^{\infty}\left(\mathbb{R}^{N}\right)^{m}$ is equipped with the norm $|\cdot|_{\infty} ; H^{1}\left(\mathbb{R}^{N}\right)^{m}$ and $H^{2}\left(\mathbb{R}^{N}\right)^{m}$ are the usual Sobolev spaces, equipped with the norms $|\cdot|_{H^{1}}$ and $|\cdot|_{H^{2}}$. Finally, the space $\mathbf{L}_{\mathbf{1}}$ denotes the subspace of $\mathbf{L}$ made out of functions which belong to $C^{4}\left(\mathbb{R}^{N}\right)^{m}$ whose first four derivatives are bounded. In this article, we use the classical multi-index notation: if $\alpha=\left(\alpha_{1}, \ldots, \alpha_{n}\right) \in \mathbb{N}^{n}$, $|\alpha|=\alpha_{1}+\ldots+\alpha_{n}$ and $\partial^{\alpha}=\partial_{1}^{\alpha_{1}} \ldots \partial_{n}^{\alpha_{n}}$. The main result is Theorem 4.11 which can be stated as follows: for all $u_{0}$ in $\mathbf{L}_{1}$ and for all $\tau>0$, there exists $C$ and $h_{0}$ such that for all $h \in\left(0, h_{0}\right]$, for all $n$ such that $n h \leq \tau$

$$
\left|\left(W^{h}\right)^{n} u_{0}-T^{n h} u_{0}\right|_{H} \leq C h|\ln h|\left|u_{0}\right|_{H} .
$$

We observe that this result is slightly different from the one obtained in [2]: here we work with a rather general system of reaction-diffusion but with stronger assumptions on the initial data; in [2], only a scalar reaction-diffusion equation was considered, but $L^{\infty}$ estimates were obtained. More precisely, for a scalar reactiondiffusion equation of the form

$$
\begin{cases}\frac{\partial u}{\partial t}-\frac{\partial^{2} u}{\partial x^{2}}+f(u)=0, & x \in \mathbb{R}, t>0, \\ u(0, x)=u_{0}(x), & x \in \mathbb{R},\end{cases}
$$

with $f$ a Lipschitz continuous function belonging to $C^{3}(\mathbb{R})$ with bounded derivatives and $u_{0}$ a continuous function bounded over $\mathbb{R}$, we have shown that the difference between $\left(W^{h}\right)^{n} u_{0}$ and $T^{n h} u_{0}$ in $L^{\infty}$-norm is in $O(\sqrt{h})$.

This article is organized as follows. In Section 2, we prove some results on the operators $-M \Delta$ and $X^{t}=e^{t M \Delta}$. In Section 3, we consider the linear case of (1.1), where $F(u)=V u$ and $V$ is bounded; in this case the explicit solution of (1.1) is given by $e^{t(M \Delta-V)} u_{0}$. We deduce from properties of sectorial operators an estimate on the difference between $e^{t(M \Delta-V)}$ and $Z^{t}=e^{t M \Delta / 2} e^{-t V} e^{t M \Delta / 2}$ in operator norms $\mathcal{L}(H)$ and $\mathcal{L}\left(H^{2}, H\right)$. In Section 4, we prove Theorem 4.11 with the help of a comparison with the linear case. 
Remark 1.1. Theorem 4.11 remains true if $F$ depends also on the space variable. We suppose that $F=F(u)$ for simplicity.

\section{SoMe PROPERTIES OF THE OPERATOR-M}

In this section, we begin by recalling the definition of sectorial operators, as given by Henry [10]. For $\theta$ in $(0, \pi / 2)$ and real $a$, we denote $S_{a, \theta}$ the subset of $\mathbb{C}$ defined by

$$
S_{a, \theta}=\{\zeta \in \mathbb{C}: \theta \leq|\arg (\zeta-a)| \leq \pi \text { and } \zeta \neq a\} .
$$

Definition 2.1. A linear operator $C$ in $H$ is called sectorial if it is closed, densely defined, and if there exist $\theta, a$ and $M_{0} \geq 1$ such that $S_{a, \theta}$ is included in $\rho(C)$, the resolvent set of $C$, and such that for all $\zeta$ in $S_{a, \theta}$

$$
\left|(\zeta-C)^{-1}\right|_{\mathcal{L}(H)} \leq M_{0} /|\zeta-a| .
$$

For any $\phi$ in $(\theta, \pi / 2)$, we denote by $\Gamma$ a contour in $S_{a, \theta}$, which verifies $\Gamma(-s)=$ $\overline{\Gamma(s)}$. Let us define $D_{\phi}$ by

$$
D_{\phi}: s \rightarrow s e^{i \phi},
$$

and we also request $\Gamma$ to be asymptotic to $D_{\phi}$ as $s \rightarrow+\infty$ (see Figure 1).

From Henry [10, we know that if $C$ is a sectorial operator in $H$, then $-C$ is the infinitesimal generator of an analytic semigroup $\left\{e^{-t C}\right\}_{t \geq 0}$, where

$$
e^{-t C}=\frac{1}{2 \pi i} \int_{\Gamma} e^{-t \zeta}(\zeta-C)^{-1} d \zeta
$$

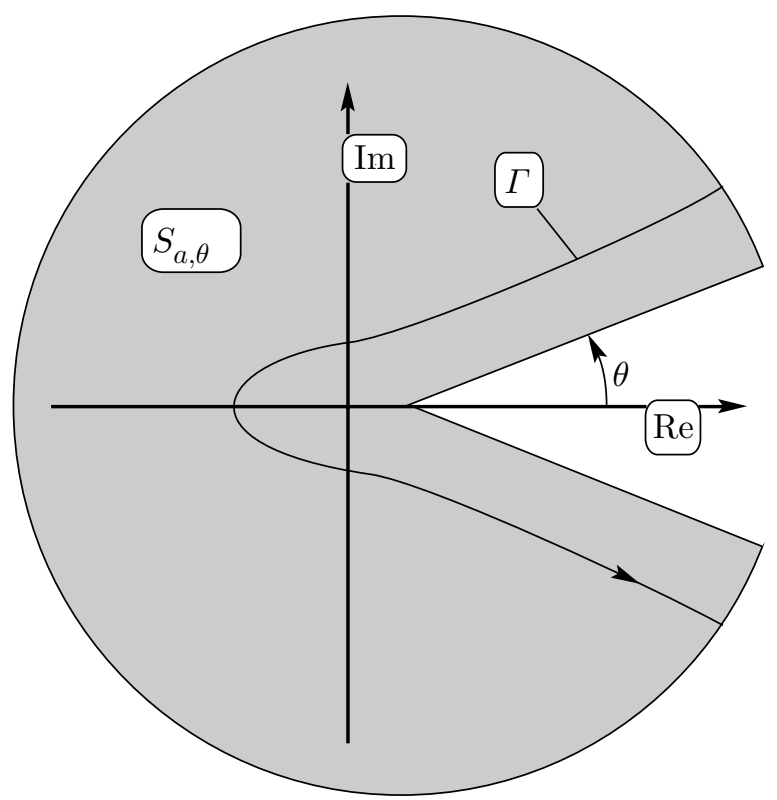

Figure 1. The path $\Gamma$ in the complex plane. 
Given an $m \times m$ matrix $M$ whose spectrum is included in $\{\mathfrak{R} z>0\}$, we define the operator $A$ by

$$
D(A)=H^{2}\left(\mathbb{R}^{N}\right)^{m}, \quad A=-M \Delta
$$

and we have the following result.

Theorem 2.2. The operator $A$ is sectorial in $H$.

The proof depends on the following lemma.

Lemma 2.3. There exist $\theta_{0} \in(0, \pi / 2)$ and a constant $C>0$ such that, for all $\zeta \in S_{0, \theta_{0}}$ :

$$
\sup _{t \geq 0}\left|(\zeta-t M)^{-1}\right|_{\mathcal{B}\left(\mathbb{C}^{m}\right)} \leq C /|\zeta|
$$

and

$$
\sup _{t \geq 0}\left|t(\zeta-t M)^{-1}\right|_{\mathcal{B}\left(\mathbb{C}^{m}\right)} \leq C
$$

Proof. Let us denote by $\mu_{1}$ an eigenvalue of $M$ such that $\left|\arg \mu_{1}\right| \geq|\arg \mu|$ for all $\mu \in \sigma(M)$. We introduce $\varepsilon>0$ such that $\left|\arg \mu_{1}\right|+\varepsilon<\pi / 2$, and we choose $\theta_{0}=\left|\arg \mu_{1}\right|+\varepsilon$. that

We now suppose that $\zeta$ belongs to $S_{0, \theta_{0}}$ and we consider $\omega$ and $\psi$ in $\mathbb{R}^{m}$ such

$$
(\zeta-t M) \omega=\psi
$$

Our purpose is to show that there exists a constant $C>0$ such that $|\omega| \leq C|\psi| /|\zeta|$. We assume that $\omega \neq 0$, we take the scalar product in the complexified space $\mathbb{C}^{m}$ with $\omega$, and we take the imaginary and the real part of this scalar product to obtain

$$
|\Im \zeta||\omega| \leq|\psi|
$$

and

$$
\mathfrak{R} \zeta \omega \cdot \omega-t(S M \omega \mid \omega)=\mathfrak{R}(\psi \cdot \omega) .
$$

We infer from (1.9) and 2.7 that

$$
(\mathfrak{R} \zeta-t \beta)|\omega| \geq-|\psi| .
$$

If $\Re \zeta<0$, we deduce from (2.8) that

$$
|\mathfrak{R} \zeta||\omega| \leq|\psi|
$$

We add the square of (2.6) and (2.9) and we find

$$
|\omega| \leq \frac{\sqrt{2}|\psi|}{|\zeta|}
$$

If $\Re \zeta>0$, then $|\Im \zeta| \geq|\zeta| \sin \theta_{0}$, and we infer from (2.6) that

$$
|\omega| \leq \frac{|\psi|}{|\zeta| \sin \theta_{0}}
$$

Therefore (2.3) is established with $C=\max \left(\sqrt{2}, 1 / \sin \theta_{0}\right)$. Finally, we deduce (2.4) from (2.3) and the triangle inequality. 
Proof of Theorem 2.2, Let $u$ belong to $H$ and let

$$
\mathcal{F}(u)(\xi)=\int_{\mathbb{R}^{N}} u(x) e^{-2 \pi i\langle x \cdot \xi\rangle} d x
$$

be its Fourier transform; here $\langle x . \xi\rangle$ is the duality product of $x$ and $\xi$. It is a well known fact that

$$
\mathcal{F}(\Delta u)(\xi)=-4 \pi^{2}|\xi|^{2} \mathcal{F}(u)(\xi)
$$

For $\theta_{0}$ defined in Lemma 2.3, we deduce that the resolvent set of $A$ is included in $S_{0, \theta_{0}}$. Moreover, for all $\zeta \in S_{0, \theta_{0}}$, we have

$$
\mathcal{F}\left(\left((\zeta+M \Delta)^{-1}\right) u\right)(\xi)=\left(\zeta-4 \pi^{2}|\xi|^{2} M\right)^{-1} \mathcal{F}(u)(\xi),
$$

and it follows from (2.3) that

$$
\left|(\zeta+M \Delta)^{-1}\right|_{\mathcal{L}(H)} \leq C /|\zeta| .
$$

This concludes the proof of Theorem 2.2

We will need also estimates on $\partial_{i}(\zeta+M \Delta)^{-1}$ and $\partial_{i j}(\zeta+M \Delta)^{-1}$.

Lemma 2.4. There exists a constant $C>0$ such that for all $i$ and $j$ in $\{1, \ldots, n\}$, for all $\zeta \in S_{0, \theta_{0}}$, and for all $u \in H$, we have

$$
\left|\partial_{i}(\zeta+M \Delta)^{-1} u\right|_{H} \leq \frac{C}{\sqrt{|\zeta|}}|u|_{H} \quad \text { and } \quad\left|\partial_{i j}(\zeta+M \Delta)^{-1} u\right|_{H} \leq C|u|_{H} .
$$

Proof. Since

$$
\left|\mathcal{F}\left(\partial_{i j}(\zeta+M \Delta)^{-1} u\right)\right|_{H} \leq \sup _{\xi \in \mathbb{R}^{N}}\left(|\xi|^{2}\left|\left(\zeta-|\xi|^{2} M\right)^{-1}\right|_{\mathcal{B}\left(\mathbb{C}^{m}\right)}\right)|u|_{H},
$$

the second inequality of (2.10) is a consequence of inequality (2.4). For $u$ in $H$, we have

$$
\begin{aligned}
\left|\nabla(\zeta+M \Delta)^{-1} u\right|_{H}^{2} & \leq\left|\Delta(\zeta+M \Delta)^{-1} u\right|_{H}\left|(\zeta+M \Delta)^{-1} u\right|_{H} \\
& \leq \frac{C}{|\zeta|}|u|_{H}^{2}
\end{aligned}
$$

and this proves the first inequality of (2.10).

We now prove the regularizing effect of the operator $X^{t}=e^{t M \Delta}$.

Lemma 2.5. There exists a constant $C>0$, such that, for all $u \in H$, all $j \in$ $\{1, \ldots, n\}$ and all $t>0$, the following inequality holds:

$$
\left|\partial_{j}\left(e^{t M \Delta} u\right)\right|_{H} \leq \frac{C}{\sqrt{t}}|u|_{H} .
$$

Proof. Since, for all $j \in\{1, \ldots, n\}$

$$
\mathcal{F}\left(\partial_{j}\left(e^{t M \Delta} u\right)\right)(\xi)=-2 \pi i \xi_{j} e^{-4 \pi^{2} M t|\xi|^{2}} \mathcal{F}(u)(\xi),
$$

it is sufficient to show that there exists a constant $C>0$, such that

$$
\sup _{\xi \in \mathbb{R}^{m}}|| \xi\left|e^{-t M|\xi|^{2}}\right|_{\mathcal{B}\left(\mathbb{R}^{m}\right)} \leq \frac{C}{\sqrt{t}} .
$$

If we let $\sqrt{t} \xi=\eta$, it is also sufficient to see that

$$
\sup _{\eta \in \mathbb{R}^{m}}|| \eta\left|e^{-M|\eta|^{2}}\right|_{\mathcal{B}\left(\mathbb{R}^{m}\right)} \leq C .
$$


For $\psi$ in $\mathbb{R}^{m}$ and $s \geq 0$, denote $\gamma(s)=e^{-s M} \psi$. It follows from (1.9) that $\gamma(s)$ verifies

$$
\frac{1}{2} \frac{d}{d s}|\gamma(s)|^{2}+\beta|\gamma(s)|^{2} \leq 0
$$

thus

$$
|\gamma(s)|^{2} \leq e^{-2 \beta s}|\psi|^{2}
$$

and we infer that

$$
\sup _{\eta \in \mathbb{R}^{m}}|| \eta\left|e^{-M|\eta|^{2}}\right|_{\mathcal{B}\left(\mathbb{R}^{m}\right)} \leq \sup _{\eta \in \mathbb{R}^{m}}\left(|\eta| e^{-\beta|\eta|^{2}}\right) \leq C .
$$

This concludes the proof of Lemma 2.5.

Finally, we give the following result, which can be deduced from Theorem 1.4.3, page 26 of Henry [10]:

Lemma 2.6. Let $u$ belong to $H^{2}\left(\mathbb{R}^{N}\right)^{m}$. There exists a constant $C>0$ such that for all $t>0$

$$
\left|e^{t M \Delta} u-u\right|_{H} \leq C t|u|_{H^{2}}
$$

\section{Estimates ON THE LinEAR CASE}

In this section, we consider the linear case of (1.1); more precisely, we consider the equation

$$
\begin{cases}\frac{\partial u}{\partial t}-M \Delta u+V u=0, & x \in \mathbb{R}^{N}, t>0, \\ u(0, x)=u_{0}(x), & x \in \mathbb{R}^{N},\end{cases}
$$

where $V$ is a bounded function. We deduce from a representation formula of $Z^{2 t}=e^{t M \Delta} e^{-2 t V} e^{t M \Delta}$ an estimate on the difference between $e^{2 t(M \Delta-V)}$ and $e^{t M \Delta} e^{-2 t V} e^{t M \Delta}$. We now introduce some classes of functions $V$.

Definition 3.1. We denote $\mathcal{V}^{4}(c)$ the set of functions $V$ of class $C^{4}$ such that there exists $c>0$ such that for all $x \in \mathbb{R}^{N}$ and all $\alpha \in \mathbb{N}^{n}$ with $0 \leq|\alpha| \leq 4$,

$$
\left|\partial^{\alpha} V(x)\right| \leq c
$$

and we denote $\mathcal{V}^{\infty}(c)$ the set of functions $V$ of class $C^{\infty}$ belonging to $\mathcal{V}^{4}(c)$ and such that $\partial^{\alpha} V$ is of slow growth for $|\alpha| \geq 5$.

Let $V_{i j}, 1 \leq i, j \leq m$, be $m^{2}$ elements of $\mathcal{V}^{\infty}(c)$ and let us denote $V$ by the matrix

$$
V=\left(V_{i j}\right)_{1 \leq i, j \leq m}
$$

With a slight abuse of notation, we will say that $V$ belongs also to $\mathcal{V}^{\infty}(c)$. We identify $V$ and the matrix multiplication by $V$ and introduce the operator $B$ defined by

$$
D(B)=H, \quad B=V
$$

It follows from Definition 3.1 that $B$ is bounded in $H$ and thus sectorial in $H$. 
3.1. A representation formula for $Z^{2 t}$. We assume that $V$ belongs to $\mathcal{V}^{\infty}(c)$. We have shown that the operator $A=-M \Delta$ and $B=V$ are sectorial. Without loss of generality, we suppose that the number $a$ of Definition 2.1 vanishes also for the second operator

$$
a=0 .
$$

This assumption will be dropped at the end of this part.

Let $H_{0}$ be the Schwartz space $\mathcal{S}\left(\mathbb{R}^{N}\right)^{m}$, which is a dense subspace of $H$. We notice that $A H_{0}$ and $B H_{0}$ are included in $H_{0}$. Moreover, for all $z$ in $\rho(A)$ (resp. in $\rho(B)),(z-A)^{-1} H_{0}$ (resp. $\left.(z-B)^{-1} H_{0}\right)$ are included in $H_{0}$. For $\zeta \in \mathbb{C}^{3}$, $\zeta=\left(\zeta_{1}, \zeta_{2}, \zeta_{3}\right)$, we let $l \zeta=\zeta_{1}+2 \zeta_{2}+\zeta_{3}$. It follows from (2.2) that, for all $u \in H_{0}$, we have the representation

$$
Z^{2 t} u=\frac{1}{(2 \pi i)^{3}} \int_{\Gamma_{1} \times \Gamma_{2} \times \Gamma_{3}} e^{-t l \zeta}\left(\zeta_{1}+M \Delta\right)^{-1}\left(\zeta_{2}-V\right)^{-1}\left(\zeta_{3}+M \Delta\right)^{-1} u d \zeta,
$$

where $\Gamma_{1}, \Gamma_{2}, \Gamma_{3}$ are paths like in Figure 1. We also have the following result.

Lemma 3.2 (Dia and Schatzman [3]). For all $u \in H_{0}$, we have

$$
\frac{d}{d t} Z^{2 t} u+2(V-M \Delta) Z^{2 t} u=R(t) u
$$

where

$$
R(t) u=\frac{1}{(2 \pi i)^{3}} \int_{\Gamma_{1} \times \Gamma_{2} \times \Gamma_{3}} e^{-t l \zeta} F_{0}(\zeta) u d \zeta
$$

and $F_{0}(\zeta)$ is defined by

$$
\begin{aligned}
F_{0}(\zeta) u= & -2\left(\zeta_{1}+M \Delta\right)^{-1} \gamma_{3}\left(\zeta_{1}+M \Delta\right)^{-2}\left(\zeta_{2}-V\right)^{-1}\left(\zeta_{3}+M \Delta\right)^{-1} u \\
& -\left(\zeta_{1}+M \Delta\right)^{-1}\left(\zeta_{2}-V\right)^{-1} \gamma_{2}\left(\zeta_{2}-V\right)^{-2}\left(\zeta_{3}+M \Delta\right)^{-1} u \\
& +\left(\zeta_{1}+M \Delta\right)^{-1} \gamma_{3}\left(\zeta_{1}+M \Delta\right)^{-1}\left(\zeta_{2}-V\right)^{-2}\left(\zeta_{3}+M \Delta\right)^{-1} u,
\end{aligned}
$$

with

$$
\gamma_{2}=[V,[V, M \Delta]], \gamma_{3}=[M \Delta,[M \Delta, V]]
$$

3.2. Applications of the representation formula. We infer from (3.3) and Duhamel's formula that for all $u$ in $H_{0}$

$$
Z^{2 t} u=e^{2 t(M \Delta-V)} u+\int_{0}^{t} e^{2(t-s)(M \Delta-V)} R(s) u d s .
$$

The following theorem enables us to estimate the function $R(t) u$. We recall that $\mathcal{V}(c)$ has been defined at Definition 3.1

Theorem 3.3. There exists a constant $C(c)$ such that for all $V \in \mathcal{V}^{\infty}(c)$ satisfying (3.2) and all $u \in H_{0}$, the following estimate holds:

$$
\forall t \in] 0,1], \quad|R(t) u|_{H} \leq C(c)|u|_{H} .
$$

Remark 3.4. The principle of the proof is identical to the principle of the proof given in [4, but the result is different since the commutators are of higher order. The difficulty is seen in the analysis of (3.28) and (3.33) below. Thus, instead of an estimate $|R(t)|_{\mathcal{L}(H)}=O(t)$, we find an estimate $|R(t)|_{\mathcal{L}(H)}=O(1)$. 
We will prove Theorem 3.3 in several steps. We perform the change of variable $\left(z_{1}, z_{2}, z_{3}\right)=t\left(\zeta_{1}, \zeta_{2}, \zeta_{3}\right)$ and obtain

$$
R(t) u=\frac{1}{(2 \pi i)^{3}} \int_{t \Gamma_{1} \times t \Gamma_{2} \times t \Gamma_{3}} e^{-l z} F_{0}(z / t) u \frac{d z}{t^{3}} .
$$

Thanks to Cauchy's Theorem, we can rewrite (3.9) under the form

$$
R(t) u=\frac{1}{(2 \pi i)^{3}} \int_{\Gamma_{1} \times \Gamma_{2} \times \Gamma_{3}} e^{-l z} F_{0}(z / t) u \frac{d z}{t^{3}} .
$$

Using the decomposition of $F_{0}$ given in (3.5), we write

$$
F_{0}\left(\frac{z}{t}\right)=P_{1}\left(\frac{z}{t}\right) u+P_{2}\left(\frac{z}{t}\right) u+P_{3}\left(\frac{z}{t}\right) u,
$$

where

$$
P_{1}\left(\frac{z}{t}\right)=-2\left(\frac{z_{1}}{t}+M \Delta\right)^{-1} \gamma_{3}\left(\frac{z_{1}}{t}+M \Delta\right)^{-2}\left(\frac{z_{2}}{t}-V\right)^{-1}\left(\frac{z_{3}}{t}+M \Delta\right)^{-1},
$$

$$
P_{2}\left(\frac{z}{t}\right)=-\left(\frac{z_{1}}{t}+M \Delta\right)^{-1}\left(\frac{z_{2}}{t}-V\right)^{-1} \gamma_{2}\left(\frac{z_{2}}{t}-V\right)^{-2}\left(\frac{z_{3}}{t}+M \Delta\right)^{-1},
$$

$$
P_{3}\left(\frac{z}{t}\right)=\left(\frac{z_{1}}{t}+M \Delta\right)^{-1} \gamma_{3}\left(\frac{z_{1}}{t}+M \Delta\right)^{-1}\left(\frac{z_{2}}{t}-V\right)^{-2}\left(\frac{z_{3}}{t}+M \Delta\right)^{-1} .
$$

Without loss of generality, we assume that, for all $z \in \Gamma_{1} \times \Gamma_{2} \times \Gamma_{3}$,

$$
\left|z_{i}\right| \geq 1, \quad i=1,2,3 .
$$

This relation leads to a simplification of the proofs in [4. The proof of Theorem 3.3 depends now on two lemmas:

Lemma 3.5. There exists a constant $C_{2}(c)$ such that, for all $\left.\left.t \in\right] 0,1\right], z \in \Gamma_{1} \times$ $\Gamma_{2} \times \Gamma_{3}$ and $u \in H_{0}$

$$
\left|P_{2}(z / t) u\right|_{H} \leq C_{2}(c) t^{4}|u|_{H}
$$

Proof. We calculate the commutator $\gamma_{2}$ appearing in (3.12). For all $\alpha \in \mathbb{N}^{n}$ such that $|\alpha| \leq 4$, let us define the matrices $\partial_{\alpha} V$ by $\partial_{\alpha} V=\left(\partial_{\alpha} V_{i j}\right)_{1 \leq i, j \leq m}$. It is clear that

$$
\begin{gathered}
{[\Delta, V]=\Delta V+2 \sum_{i=1}^{n}\left(\partial_{i} V\right) \partial_{i}} \\
{[[\Delta, V], V]=[\Delta V, V]+2 \sum_{i=1}^{n}\left[\partial_{i} V, V\right] \partial_{i}+2 \sum_{i=1}^{n}\left(\partial_{i} V\right)^{2} .}
\end{gathered}
$$

Since

$$
\gamma_{2}=[V,[V, M \Delta]]=[[M \Delta, V], V]=M[[\Delta, V], V]+2[M, V][\Delta, V]+[[M, V], V] \Delta,
$$


we deduce from (3.15) and (3.16) that

$$
\begin{aligned}
\gamma_{2}= & M[\Delta V, V]+2 \sum_{i=1}^{n} M\left(\partial_{i} V\right)^{2}+2[M, V] \Delta V \\
& +2 \sum_{i=1}^{n}\left(M\left[\partial_{i} V, V\right]+2[M, V] \partial_{i} V\right) \partial_{i}+[[M, V], V] \Delta .
\end{aligned}
$$

The important piece of this decomposition of $\gamma_{2}$ is the last one: it would vanish in the scalar case, the fact that we have a system makes the problem more difficult. We infer from this decomposition that there exist bounded functions $N$ and $N_{i}$, $i \in\{1, \ldots, n\}$, depending only on $M$ and the first two derivatives of $V$ such that

$$
\gamma_{2}=N+\sum_{i=1}^{n} N_{i} \partial_{i}+[[M, V], V] \Delta .
$$

We use this decomposition to estimate $P_{2}$. We infer from (2.1) and (3.14) that

$$
\left|N\left(\frac{z_{2}}{t}-V\right)^{-2}\left(\frac{z_{3}}{t}+M \Delta\right)^{-1} u\right|_{H} \leq C(c) t^{3}|u|_{H} .
$$

We now have to estimate $\partial_{i}\left(z_{2} / t-V\right)^{-2}\left(z_{3} / t+M \Delta\right)^{-1} u$. We recall that for general operators $C, D$ and $\zeta$ in $\mathbb{C}$, we have

$$
C(\zeta-D)^{-1}=(\zeta-D)^{-1} C+(\zeta-D)^{-1}[C, D](\zeta-D)^{-1} .
$$

Since $\left[\partial_{i}, V\right]=\partial_{i} V$, we deduce the following relations:

$$
\begin{aligned}
\partial_{i}\left(\frac{z_{2}}{t}-V\right)^{-1}= & \left(\frac{z_{2}}{t}-V\right)^{-1} \partial_{i}+\left(\frac{z_{2}}{t}-V\right)^{-1} \partial_{i} V\left(\frac{z_{2}}{t}-V\right)^{-1}, \\
\partial_{i}\left(\frac{z_{2}}{t}-V\right)^{-2}= & \left(\frac{z_{2}}{t}-V\right)^{-2} \partial_{i}+\left(\frac{z_{2}}{t}-V\right)^{-2} \partial_{i} V\left(\frac{z_{2}}{t}-V\right)^{-1} \\
& +\left(\frac{z_{2}}{t}-V\right)^{-1} \partial_{i} V\left(\frac{z_{2}}{t}-V\right)^{-2} .
\end{aligned}
$$

We infer from Lemma 2.4 that for all $i, i$ in $\{1, \ldots, n\}$,

$$
\left|\partial_{i}\left(\frac{z_{3}}{t}+M \Delta\right)^{-1} u\right|_{H} \leq C(c) \sqrt{t}|u|_{H} ;
$$

thus we deduce from (3.21) that

$$
\left|N_{i} \partial_{i}\left(\frac{z_{2}}{t}-V\right)^{-2}\left(\frac{z_{3}}{t}+M \Delta\right)^{-1} u\right|_{H} \leq C(c)\left(t^{4}+t^{5 / 2}\right)|u|_{H},
$$

and so

$$
\left|N_{i} \partial_{i}\left(\frac{z_{2}}{t}-V\right)^{-2}\left(\frac{z_{3}}{t}+M \Delta\right)^{-1} u\right|_{H} \leq C(c) t^{5 / 2}|u|_{H} .
$$

Finally, we have to estimate $\Delta\left(z_{2} / t-V\right)^{-2}\left(z_{3} / t+M \Delta\right)^{-1} u$, we deduce from (3.19) that

$$
\begin{aligned}
\Delta\left(\frac{z_{2}}{t}-V\right)^{-2}= & \left(\frac{z_{2}}{t}-V\right)^{-2} \Delta+\left(\frac{z_{2}}{t}-V\right)^{-2}[\Delta, V]\left(\frac{z_{2}}{t}-V\right)^{-1} \\
& +\left(\frac{z_{2}}{t}-V\right)^{-1}[\Delta, V]\left(\frac{z_{2}}{t}-V\right)^{-2} .
\end{aligned}
$$


We infer from (2.1) and Lemma 2.4 that

$$
\left|\left(\frac{z_{2}}{t}-V\right)^{-2} \Delta\left(\frac{z_{3}}{t}+M \Delta\right)^{-1} u\right|_{H} \leq C(c) t^{2}|u|_{H} .
$$

Let us denote

$$
Q_{1}\left(\frac{z}{t}\right)=\left(\frac{z_{2}}{t}-V\right)^{-2}[\Delta, V]\left(\frac{z_{2}}{t}-V\right)^{-1}+\left(\frac{z_{2}}{t}-V\right)^{-1}[\Delta, V]\left(\frac{z_{2}}{t}-V\right)^{-2} .
$$

We deduce from relation (3.15), decompositions (3.20) and (3.22) that

$$
\left|Q_{1}\left(\frac{z}{t}\right)\left(\frac{z_{3}}{t}+M \Delta\right)^{-1} u\right|_{H} \leq C(c) t^{3} \sqrt{t}|u|_{H} .
$$

It follows from (3.17) and estimates (3.18), (3.22), (3.24), (3.25) that there exists $C(c)$ such that for all $t \in] 0,1], z \in \Gamma_{1} \times \Gamma_{2} \times \Gamma_{3}$ and $u \in H_{0}$. We have

$$
\left|\gamma_{2}\left(\frac{z_{2}}{t}-V\right)^{-2}\left(\frac{z_{3}}{t}+M \Delta\right)^{-1} u\right|_{H} \leq C(c) t^{2}|u|_{H}
$$

We now deduce from (2.1) and the definition of $P_{2}$ that there exists $C_{2}(c)$ such that

$$
\left|P_{2}\left(\frac{z}{t}\right) u\right|_{H} \leq C_{2}(c) t^{4}|u|_{H}
$$

This concludes the proof of Lemma 3.5.

Lemma 3.6. For $j \in\{1,3\}$, there exists a constant $C_{j}(c)$ such that, for all $t \in$ ]0,1], $z \in \Gamma_{1} \times \Gamma_{2} \times \Gamma_{3}$ and $u \in H_{0}$,

$$
\left|P_{j}(z / t) u\right|_{H} \leq C_{j}(c) t^{3}|u|_{H} .
$$

Proof. We first develop the commutator

$$
\gamma_{3}=M^{2}[\Delta,[\Delta, V]]+M[M,[\Delta, V]] \Delta+M[\Delta,[M, V]] \Delta+[M,[M, V]] \Delta^{2} .
$$

We notice that this operator involves generally a bilaplacian, as soon as the problem under consideration is not scalar. We begin with the case $j=1$, let us define

$$
\begin{aligned}
Q_{1}\left(\frac{z}{t}\right) u= & \left(\frac{z_{1}}{t}+M \Delta\right)^{-1}[M,[M, V]] \Delta^{2}\left(\frac{z_{1}}{t}+M \Delta\right)^{-2}\left(\frac{z_{2}}{t}-V\right)^{-1} \\
& \times\left(\frac{z_{3}}{t}+M \Delta\right)^{-1} u .
\end{aligned}
$$

We infer from Lemma 2.4 and (2.1) that

$$
\left|Q_{1}(z / t) u\right|_{H} \leq C(c) t^{3}|u|_{H} .
$$

Consider now the operator $L$ defined by

$$
L=M^{2}[\Delta,[\Delta, V]]+M[M,[\Delta, V]] \Delta+M[\Delta,[M, V]] \Delta .
$$

Using (3.15), we deduce that there exist bounded functions $N, N_{i}^{0}, N_{i}^{1}$, i in $\{1, \ldots, n\}$ and $N_{i j}, i$ and $j$ in $\{1, \ldots, n\}$, depending only on $M$ and the first four derivatives of $V$ such that

$$
L=N+\sum_{1 \leq i \leq n} N_{i}^{0} \partial_{i}+\sum_{1 \leq i, j \leq n} N_{i j} \partial_{i j}+\sum_{1 \leq i \leq n} N_{i}^{1} \partial_{i} \Delta .
$$

We infer from (2.1) and Lemma 2.4 that

$$
\left|\left(\frac{z_{1}}{t}+M \Delta\right)^{-1} L\left(\frac{z_{1}}{t}+M \Delta\right)^{-2}\left(\frac{z_{2}}{t}-V\right)^{-1}\left(\frac{z_{3}}{t}+M \Delta\right)^{-1} u\right|_{H} \leq C(c) t^{3} \sqrt{t}|u|_{H} .
$$


Relation (3.26) concerning $P_{1}$ is now a consequence of (3.28) and (3.30).

There remains to consider the case of $P_{3}$. The proof is very similar: we first study the function

$$
\begin{aligned}
Q_{2}\left(\frac{z}{t}\right) u= & \left(\frac{z_{1}}{t}+M \Delta\right)^{-1}[M,[M, V]] \Delta^{2}\left(\frac{z_{1}}{t}+M \Delta\right)^{-1}\left(\frac{z_{2}}{t}-V\right)^{-2} \\
& \times\left(\frac{z_{3}}{t}+M \Delta\right)^{-1} u,
\end{aligned}
$$

that we rewrite under the form

$$
\begin{aligned}
Q_{2}\left(\frac{z}{t}\right) u= & \left(\frac{z_{1}}{t}+M \Delta\right)^{-1}[M,[M, V]] \Delta\left(\frac{z_{1}}{t}+M \Delta\right)^{-1} \Delta\left(\frac{z_{2}}{t}-V\right)^{-2} \\
& \times\left(\frac{z_{3}}{t}+M \Delta\right)^{-1} u .
\end{aligned}
$$

It follows from (3.23) that $Q_{2}$ admits the decomposition $Q_{2}(z / t)=Q_{3}(z / t)+$ $Q_{4}(z / t)$, with

$$
\begin{aligned}
Q_{3}\left(\frac{z}{t}\right) u= & \left(\frac{z_{1}}{t}+M \Delta\right)^{-1}[M,[M, V]] \Delta\left(\frac{z_{1}}{t}+M \Delta\right)^{-1}\left(\frac{z_{2}}{t}-V\right)^{-2} \\
& \times \Delta\left(\frac{z_{3}}{t}+M \Delta\right)^{-1} u
\end{aligned}
$$

and $Q_{4}(z / t)$ is a function which can be estimated thanks to (3.25) by

$$
\left|Q_{4}(z / t) u\right|_{H} \leq C(c) t^{4} \sqrt{t}|u|_{H}
$$

We also notice that

$$
\left|Q_{3}(z / t) u\right|_{H} \leq C t^{3}|u|_{H}
$$

We return to the decomposition of $L$ given in (3.29) and let

$$
L=L_{1}+L_{2}
$$

with

$$
L_{1}=N+\sum_{1 \leq i \leq n} N_{i}^{0} \partial_{i}+\sum_{1 \leq i, j \leq n} N_{i j} \partial_{i j} \quad \text { and } \quad L_{2}=\sum_{1 \leq i \leq n} N_{i}^{1} \partial_{i} \Delta .
$$

We infer from (2.1) and Lemma 2.4 that

$$
\left|\left(\frac{z_{1}}{t}+M \Delta\right)^{-1} L_{1}\left(\frac{z_{1}}{t}+M \Delta\right)^{-1}\left(\frac{z_{2}}{t}-V\right)^{-2}\left(\frac{z_{3}}{t}+M \Delta\right)^{-1} u\right|_{H} \leq C(c) t^{4}|u|_{H},
$$

and deduce from (3.22) that

$$
\left|\left(\frac{z_{1}}{t}+M \Delta\right)^{-1} L_{2}\left(\frac{z_{1}}{t}+M \Delta\right)^{-1}\left(\frac{z_{2}}{t}-V\right)^{-2}\left(\frac{z_{3}}{t}+M \Delta\right)^{-1} u\right|_{H} \leq C(c) t^{7 / 2}|u|_{H} .
$$

Relation (3.26) for $P_{3}$ is now a consequence of (3.32), (3.33), (3.34) and (3.35).

Proof of Theorem 3.3. It follows from Lemma 3.5 and Lemma 3.6 that there exists a constant such that, for all $t \in] 0,1], z \in \Gamma_{1} \times \Gamma_{2} \times \Gamma_{3}$ and $u \in H_{0}$,

$$
|F(z / t) u|_{H} \leq C(c) t^{3}|u|_{H},
$$

and with the help of relation (3.10) this proves (3.8).

Now we can prove the following theorem. 
Theorem 3.7. There exists a constant $C(c)>0$ such that for all elements $V$ of $\mathcal{V}^{\infty}(c)$ satisfying (3.2),

$$
\forall t \in[0,1], \quad\left|e^{t M \Delta} e^{-2 t V} e^{t M \Delta}-e^{2 t(M \Delta-V)}\right|_{\mathcal{L}(H)} \leq C(c) t .
$$

Proof. We can deduce from (3.7) that for all $u \in H_{0}$

$$
\left|Z^{2 t} u-e^{2 t(M \Delta-V)} u\right|_{H} \leq \int_{0}^{t}\left|e^{2(t-s)(M \Delta-V)} R(s) u\right|_{H} d s .
$$

Since the operator $V$ is bounded in $H$, the operator $-M \Delta+V$ is a bounded perturbation of a sectorial operator, and so is sectorial. This proves the existence of a constant $C(c)>0$ such that for all $t \in[0,1]$,

$$
\left|e^{2 t(M \Delta-V)}\right|_{\mathcal{L}(H)} \leq C(c)
$$

Then we deduce that

$$
\int_{0}^{t}\left|e^{2(t-s)(M \Delta-V)} R(s) u\right|_{H} d s \leq C(c) \int_{0}^{t}|R(s) u|_{H} d s,
$$

and thanks to Theorem 3.3 we obtain that for all $u \in H_{0}$ and for all $t \in[0,1]$

$$
\left|\left(Z^{2 t}-e^{2 t(M \Delta-V)}\right) u\right|_{H} \leq C(c) t|u|_{H}
$$

Since $H_{0}$ is dense in $H$, (3.36) holds for all $u \in H$, thus we have

$$
\left|Z^{2 t}-e^{2 t(M \Delta-V)}\right|_{\mathcal{L}(H)} \leq C(c) t
$$

The condition $V \in \mathcal{V}^{\infty}(c)$ satisfying (3.2) can be weakened:

Theorem 3.8. There exists a constant $C(c)>0$ such that for all elements $V$ of $\mathcal{V}^{4}(c)$

$$
\forall t \in[0,1], \quad\left|e^{t M \Delta} e^{-2 t V} e^{t M \Delta}-e^{2 t(M \Delta-V)}\right|_{\mathcal{L}(H)} \leq C(c) t
$$

Proof. If we suppose $a \neq 0$ and nonnegative for example, we let $U=V-a$ and we can see that

$$
e^{t M \Delta} e^{-2 t U} e^{t M \Delta}=e^{2 a t} e^{t M \Delta} e^{-t V} e^{t M \Delta} \quad \text { and } \quad e^{2 t(M \Delta-U)}=e^{2 a t} e^{2 t(\Delta-V)}
$$

so the result remains true. Finally, for $V$ in $\mathcal{V}^{4}(c)$, as in [4, we can construct a sequence $V_{m}$ belonging to $\mathcal{V}^{\infty}(c)$ which tends to $V$, and we can pass to the limit in our estimates.

The estimate of Theorem 3.8 is optimal, as is shown in the following result.

Theorem 3.9. There exists a choice of $M$ and $V$ such that there exists a constant $C>0$ such that, for small $t$,

$$
\left|e^{t M \Delta} e^{-2 t V} e^{t M \Delta}-e^{2 t(M \Delta-V)}\right|_{\mathcal{L}(H)} \geq C t
$$


Proof. We suppose that $m=2$ and choose

$$
M=\left(\begin{array}{ll}
1 & 0 \\
0 & 2
\end{array}\right), \quad V=\left(\begin{array}{ll}
0 & 1 \\
0 & 0
\end{array}\right) .
$$

Let $u$ belong to $H$ and denote $P(t) u=e^{t M \Delta} e^{-2 t V} e^{t M \Delta} u-e^{2 t(M \Delta-V)} u$. The Fourier transform of $P(t) u$ is given by

$$
\mathcal{F}(P(t) u)(\xi)=\left(e^{-4 \pi^{2} t M|\xi|^{2}} e^{-2 t V} e^{-4 \pi^{2} t M|\xi|^{2}}-e^{-8 \pi^{2} t M|\xi|^{2}-2 t V}\right) \mathcal{F}(u)(\xi) .
$$

Thus the operator norm of $P(t)$ for $t>0$ verifies

$$
|P(t)|_{\mathcal{L}(H)}=\sup _{\xi \in \mathbb{R}^{N}}\left|e^{-4 \pi^{2} t M|\xi|^{2}} e^{-2 t V} e^{-4 \pi^{2} t M|\xi|^{2}}-e^{-8 \pi^{2} t M|\xi|^{2}-2 t V}\right|_{\mathcal{B}\left(\mathbb{R}^{m}\right)} .
$$

We choose $\xi$ such that $2 \pi|\xi|=1 / \sqrt{t}$ and we deduce that

$$
|P(t)|_{\mathcal{L}(H)} \geq\left|e^{-M} e^{-2 t V} e^{-M}-e^{-2 M-2 t V}\right|_{\mathcal{B}\left(\mathbb{R}^{m}\right)} .
$$

An elementary calculation shows that

$$
e^{-M} e^{-2 t V} e^{-M}-e^{-2 M-2 t V}=t\left(\begin{array}{cc}
0 & -2 e^{-3}+e^{-2}-e^{-4} \\
0 & 0
\end{array}\right),
$$

and so our assertion is proved.

In 9], 4] and 11], which give scalar results, estimate (3.37) is replaced by an estimate $O\left(t^{1+\varepsilon}\right)$ with $\varepsilon>0$. However, in the vector case Theorem 3.9 implies that estimate (3.37) is optimal and does not suffice to prove convergence. However, it is possible to go around this difficulty by considering $Z^{2 t}-e^{2 t(M \Delta-V)}$ as an operator from $H^{2}\left(\mathbb{R}^{N}\right)^{m}$ to $L^{2}\left(\mathbb{R}^{N}\right)^{m}$. In the sequel, this loss of regularity will be compensated for by the regularizing property of $e^{t M \Delta}$.

Theorem 3.10. There exists a constant $C(c)>0$ such that for all elements $V$ of $\mathcal{V}^{4}$,

$$
\forall t \in[0,1], \quad\left|e^{t M \Delta} e^{-2 t V} e^{t M \Delta}-e^{2 t(M \Delta-V)}\right|_{\mathcal{L}\left(H^{4}, L^{2}\right)} \leq C(c) t^{2} .
$$

Proof. In a first part, we assume that $V$ belongs to $\mathcal{V}^{\infty}$ and satisfies (3.2). Let $u$ belong to $H^{2}\left(\mathbb{R}^{N}\right)^{m}$. Using the proof of Theorem 3.3 and that $|u|_{H} \leq|u|_{H^{2}}$, it is sufficient to estimate (3.27), (3.30), (3.31) and (3.35). For all, we must obtain an estimate with a power of $t$ greater than 4 instead of 3 or $7 / 2$.

We study (3.27) and (3.31); the analysis of the other expressions is quite similar and is left to the reader. Assume that $z_{3}$ belongs to $\Gamma_{3}$. Since $u \in H^{2}\left(\mathbb{R}^{N}\right)^{m}$, we infer from (2.1) that

$$
\left|\Delta\left(\frac{z_{3}}{t}+M \Delta\right)^{-1} u\right|_{H} \leq\left|\left(\frac{z_{3}}{t}+M \Delta\right)^{-1} \Delta u\right|_{H} \leq C t|u|_{H^{2}} .
$$

Thus for (3.31), we obtain

$$
\left|Q_{3}(z / t) u\right|_{H} \leq C(c) t^{4}|u|_{H^{2}} .
$$

For (3.27), we recall that

$$
\begin{aligned}
Q_{1}\left(\frac{z}{t}\right) u= & \left(\frac{z_{1}}{t}+M \Delta\right)^{-1}[M,[M, V]] \Delta^{2}\left(\frac{z_{1}}{t}+M \Delta\right)^{-2}\left(\frac{z_{2}}{t}-V\right)^{-1} \\
& \times\left(\frac{z_{3}}{t}+M \Delta\right)^{-1} u .
\end{aligned}
$$


Denote

$$
Q_{1,1}\left(\frac{z}{t}\right) u=\Delta^{2}\left(\frac{z_{1}}{t}+M \Delta\right)^{-2}\left(\frac{z_{2}}{t}-V\right)^{-1}\left(\frac{z_{3}}{t}+M \Delta\right)^{-1} u .
$$

We infer from (3.19) that

$$
\begin{aligned}
\Delta\left(\frac{z_{2}}{t}-V\right)^{-1}\left(\frac{z_{3}}{t}+M \Delta\right)^{-1} u= & \left(\frac{z_{2}}{t}-V\right)^{-1} \Delta\left(\frac{z_{3}}{t}+M \Delta\right)^{-1} u \\
& +\left(\frac{z_{2}}{t}-V\right)^{-1}[\Delta, V]\left(\frac{z_{2}}{t}-V\right)^{-1} \\
& \times\left(\frac{z_{3}}{t}+M \Delta\right)^{-1} u .
\end{aligned}
$$

Thus

$$
\left|Q_{1,1}\left(\frac{z}{t}\right) u\right|_{H} \leq C(c) t^{3}|u|_{H^{2}}
$$

and we deduce that

$$
\left|Q_{1}\left(\frac{z}{t}\right) u\right|_{H} \leq C(c) t^{4}|u|_{H^{2}}
$$

Finally, following the method of [4], Section 3 , if $V$ belongs to $\mathcal{V}^{4}$, the result remains true.

\section{Proof of convergence}

In this section, we prove the convergence of our scheme. In a first part, for $u_{0}$ in $H$, we compare $Z^{t} u_{0}$ and $T^{t} u_{0}$ in $H$-norm and deduce an estimate on $W^{t} u_{0}$ and $T^{t} u_{0}$ in $H$-norm. In a second part, we use this estimate to study $\left|\left(W^{h}\right)^{n} u_{0}-T^{n h} u_{0}\right|_{H}$. Since for all $\tau>0$ the solution of (1.1) belongs to $C([0, \tau], \mathbf{L})$, we can reduce the proof to the case where $F$ is a $C^{5}$ function with compact support and satisfying (1.2). We denote $\gamma$ a constant greater than the maximum of the first three derivatives of $F$ and such that, for all $u$ and $v$ in $H$,

$$
|F(u)-F(v)|_{H} \leq \gamma|u-v|_{H} .
$$

Since, in this case, $F$ is a Lipschitz continuous function, we begin by recalling some properties of the flows $Y^{t}$ and $T^{t}$, defined in the introduction.

4.1. Some properties of $Y^{t}$ and $T^{t}$ in the Lipschitz continuous case. We infer from Gronwall's Lemma the following result.

Lemma 4.1. There exists $\gamma$ such that for all $u_{0}$ and $v_{0}$ in $\mathbf{L}$ and all $t>0$,

$$
\left|Y^{t} u_{0}-Y^{t} v_{0}\right|_{H} \leq e^{\gamma t}\left|u_{0}-v_{0}\right|_{H} .
$$

We have the same result for the flow $T^{t}$; a proof can be found in Smoller [13] Theorem 11.15 p. 117.

Lemma 4.2. There exists $\gamma$ such that for all $u_{0}$ and $v_{0}$ in $\mathbf{L}$ and all $t>0$,

$$
\left|T^{t} u_{0}-T^{t} v_{0}\right|_{H} \leq e^{\gamma t}\left|u_{0}-v_{0}\right|_{H} .
$$

Remark 4.3. The same result holds in $L^{\infty}$-norm with $C e^{\gamma t}$ instead of $e^{\gamma t}$.

Finally we recall the regularizing effect of the flow $T^{t}$ : 
Lemma 4.4. There exists a constant $C>0$ such that for all $u_{0} \in \mathbf{L}$, all $t>0$

$$
\left|T^{t} u_{0}\right|_{H^{1}} \leq \frac{C e^{C t}}{\sqrt{t}}\left|u_{0}\right|_{H} \text { and }\left|T^{t} u_{0}\right|_{H^{2}} \leq \frac{C e^{C t}}{t}\left|u_{0}\right|_{H} .
$$

Proof. Using the regularizing effect of the operator $e^{t M \Delta}$ given in Lemma 2.5, the proof is identical to the proof of Proposition 2.1 of [6], to which the reader is referred.

4.2. Comparison of $W^{t}$ and $T^{t}$ when $F$ has a compact support. For all $u_{0}$ in $\mathbf{L}_{1}$, we denote $C\left(u_{0}\right)$ a constant depending on $\max _{0 \leq|\alpha| \leq 4}\left|\partial^{\alpha} u_{0}\right|_{\infty}$. We begin with the first result.

Theorem 4.5. For all $u_{0}$ in $\mathbf{L}_{1}$ and $t \in[0,1]$, the following estimates holds:

$$
\left|Z^{t} u_{0}-T^{t} u_{0}\right|_{H} \leq C\left(u_{0}\right) t\left|u_{0}\right|_{H}
$$

and if $u_{0}$ belongs to $H^{2}\left(\mathbb{R}^{N}\right)^{m}$

$$
\left|Z^{t} u_{0}-T^{t} u_{0}\right|_{H} \leq C\left(u_{0}\right) t^{2}\left|u_{0}\right|_{H^{2}} .
$$

We will prove Theorem 4.5 in several steps by introducing auxiliary functions. We prove estimate (4.6) using the result of Theorem 3.10, With the same method and Theorem 3.8 we can prove estimate (4.5), which is left to the reader. Thus we prove only (4.6) which is the harder of the two. We introduce the function $T_{\text {app }}^{t}\left(u_{0}\right)$ defined by the solution of the system

$$
\begin{cases}\frac{\partial v}{\partial t}-M \Delta v+D F\left(u_{0}\right) v=D F\left(u_{0}\right) u_{0}-F\left(u_{0}\right), & x \in \mathbb{R}^{N}, t>0, \\ v(0, x)=u_{0}(x), & x \in \mathbb{R}^{N} .\end{cases}
$$

We also introduce $Y_{\text {app }}^{t}\left(u_{0}, X^{t / 2} u_{0}\right)$ defined by the solution of

$$
\begin{cases}\frac{\partial w}{\partial t}+D F\left(u_{0}\right) w=D F\left(u_{0}\right) u_{0}-F\left(u_{0}\right), & x \in \mathbb{R}^{N}, t>0, \\ w(0, x)=e^{t M \Delta / 2} u_{0}(x), & x \in \mathbb{R}^{N} .\end{cases}
$$

Finally, we define $Z_{\text {app }}^{t} u_{0}$ by

$$
Z_{\mathrm{app}}^{t} u_{0}=X^{t / 2} Y_{\mathrm{app}}^{t}\left(u_{0}, X^{t / 2} u_{0}\right),
$$

and we write

$$
\begin{aligned}
Z^{t} u_{0}-T^{t} u_{0}= & Z^{t} u_{0}-Z_{\text {app }}^{t} u_{0}+Z_{\text {app }}^{t} u_{0}-T_{\text {app }}^{t} u_{0} \\
& +T_{\text {app }}^{t} u_{0}-T^{t} u_{0} .
\end{aligned}
$$

In the following sequence of lemmas, we compare the three differences appearing in the right hand side of (4.10). For simplicity we denote

$$
G\left(u_{0}\right)=D F\left(u_{0}\right) u_{0}-F\left(u_{0}\right) .
$$

Lemma 4.6. For all $u_{0}$ in $\mathbf{L}_{1}$ and $t \in[0,1]$, the following estimate holds:

$$
\left|Z_{\text {app }}^{t} u_{0}-T_{a p p}^{t} u_{0}\right|_{H} \leq C\left(u_{0}\right) t^{2}\left|u_{0}\right|_{H^{2}} .
$$

Proof. Using Duhamel's formula, the solution $v$ of (4.7) is given explicitly by

$$
v=e^{t\left(M \Delta-D F\left(u_{0}\right)\right)} u_{0}+\int_{0}^{t} e^{(t-s)\left(M \Delta-D F\left(u_{0}\right)\right)} G\left(u_{0}\right) d s,
$$


and the solution $z$ of (4.9) is given by

$$
z=e^{t M \Delta / 2}\left(e^{-t D F\left(u_{0}\right)} e^{t M \Delta / 2} u_{0}+\int_{0}^{t} e^{-(t-s) D F\left(u_{0}\right)} G\left(u_{0}\right) d s\right) .
$$

Thus, we have

$$
\begin{aligned}
v-z= & e^{t\left(M \Delta-D F\left(u_{0}\right)\right)} u_{0}-e^{t M \Delta / 2} e^{-t D F\left(u_{0}\right)} e^{t M \Delta / 2} u_{0} \\
& +\int_{0}^{t} e^{(t-s)\left(M \Delta-D F\left(u_{0}\right)\right)} G\left(u_{0}\right) d s-e^{t M \Delta / 2}\left(\int_{0}^{t} e^{-(t-s) D F\left(u_{0}\right)} G\left(u_{0}\right) d s\right)
\end{aligned}
$$

that we rewrite as

$$
\begin{aligned}
v-z= & e^{t\left(M \Delta-D F\left(u_{0}\right)\right)} u_{0}-e^{t M \Delta / 2} e^{-t D F\left(u_{0}\right)} e^{t M \Delta / 2} u_{0} \\
& +\left(1-e^{t M \Delta / 2}\right)\left(\int_{0}^{t} e^{-(t-s) D F\left(u_{0}\right)} G\left(u_{0}\right) d s\right) \\
& +\int_{0}^{t} e^{(t-s)\left(M \Delta-D F\left(u_{0}\right)\right)} G\left(u_{0}\right) d s-\int_{0}^{t} e^{-(t-s) D F\left(u_{0}\right)} G\left(u_{0}\right) d s .
\end{aligned}
$$

Using (3.39), we deduce that

$$
\left|e^{t\left(M \Delta-D F\left(u_{0}\right)\right)} u_{0}-e^{t M \Delta / 2} e^{-t D F\left(u_{0}\right)} e^{t M \Delta / 2} u_{0}\right|_{H} \leq C\left(u_{0}\right) t^{2}\left|u_{0}\right|_{H^{2}}
$$

Since $F$ has a compact support and vanishes at 0 , we have

$$
\left|G\left(u_{0}\right)\right|_{H^{2}} \leq C\left(u_{0}\right)\left|u_{0}\right|_{H^{2}}
$$

and we deduce from Lemma 2.6] that

$$
\left|\left(1-e^{t M \Delta / 2}\right)\left(\int_{0}^{t} e^{-(t-s) D F\left(u_{0}\right)} G\left(u_{0}\right) d s\right)\right|_{H} \leq C\left(u_{0}\right) t^{2}\left|u_{0}\right|_{H^{2}} .
$$

There remains to estimate the last term of the right hand side of (4.12). Since

$$
e^{t\left(M \Delta-D F\left(u_{0}\right)\right)}-e^{-t D F\left(u_{0}\right)}=e^{t\left(M \Delta-D F\left(u_{0}\right)\right)}-1+1-e^{-t D F\left(u_{0}\right)},
$$

and since the operator $M \Delta-D F\left(u_{0}\right)$ is sectorial, we infer from Lemma 2.6 and (4.14) that

$$
\left|\int_{0}^{t} e^{(t-s)\left(M \Delta-D F\left(u_{0}\right)\right)} G\left(u_{0}\right) d s-\int_{0}^{t} e^{-(t-s) D F\left(u_{0}\right)} G\left(u_{0}\right) d s\right|_{H} \leq C\left(u_{0}\right) t^{2}\left|u_{0}\right|_{H^{2}} .
$$

We can now deduce estimate (4.11) from 4.13), 4.15), 4.17). This concludes the proof of Lemma 4.6 .

Lemma 4.7. For all $u_{0}$ in $\mathbf{L}_{1}$ and $t \in[0,1]$, the following estimate holds:

$$
\left|T_{\mathrm{app}}^{t} u_{0}-T^{t} u_{0}\right|_{H} \leq C\left(u_{0}\right) t^{2}\left|u_{0}\right|_{H^{2}}
$$

Proof. For $u=T^{t} u_{0}$ and $v$ the solution of (4.7), let us define $y=v-u$. The function $y$ verifies the system

$$
\begin{cases}\frac{\partial y}{\partial t}-M \Delta y+D F\left(u_{0}\right) y=F(u)-F\left(u_{0}\right)-D F\left(u_{0}\right)\left(u-u_{0}\right), & x \in \mathbb{R}^{N}, t>0 \\ y(0, x)=0, & x \in \mathbb{R}^{N} .\end{cases}
$$


Denote $G_{0}\left(u, u_{0}\right)=F(u)-F\left(u_{0}\right)-D F\left(u_{0}\right)\left(u-u_{0}\right)$. It follows from Taylor's formula that

$$
\left|G_{0}\left(u, u_{0}\right)\right|_{H} \leq\left|\int_{0}^{1}(1-t) D^{2} F\left(u_{0}+t\left(u-u_{0}\right)\right)\left(u-u_{0}\right) \otimes\left(u-u_{0}\right) d t\right|_{H},
$$

and since $D^{2} F$ is bounded, it follows from Remark 4.3 that

$$
\left|G_{0}\left(u, u_{0}\right)\right|_{H} \leq C\left(u_{0}\right)\left|u-u_{0}\right|_{H} .
$$

Since $u-u_{0}$ is solution of

$$
u-u_{0}=\left(e^{t M \Delta}-1\right) u_{0}+\int_{0}^{t} e^{(t-s) M \Delta} F(u) d s,
$$

we deduce from the boundedness of $F$ and from Lemma 2.6 that for $t \in[0,1]$

$$
\left|u-u_{0}\right|_{H} \leq C t\left|u_{0}\right|_{H^{2}}
$$

and therefore

$$
\left|G_{0}\left(u, u_{0}\right)\right|_{H} \leq C\left(u_{0}\right) t\left|u_{0}\right|_{H^{2}} .
$$

Since the function $y=v-u$ is solution of (4.19), we obtain that

$$
|y| \leq C\left(u_{0}\right) t^{2}\left|u_{0}\right|_{H^{2}} .
$$

This concludes the proof of Lemma 4.7

Lemma 4.8. For all $u_{0}$ in $\mathbf{L}_{1}$ and $t \in[0,1]$, the following estimate holds:

$$
\left|Z_{a p p}^{t} u_{0}-Z^{t} u_{0}\right|_{H} \leq C\left(u_{0}\right) t^{2}\left|u_{0}\right|_{H^{2}} .
$$

Proof. The proof is identical to the proof of Lemma 4.7 and is left to the reader.

Proof of Theorem 4.5. We deduce from the decomposition (4.10) that relation (4.6) is a consequence of (4.11), (4.18) and (4.21).

A consequence of the previous result is the following:

Theorem 4.9. For all $u_{0}$ in $\mathbf{L}_{1}$ and $t \in[0,1]$, the following estimates holds:

$$
\left|W^{t} u_{0}-T^{t} u_{0}\right|_{H} \leq C\left(u_{0}\right) t\left(\left|u_{0}\right|_{H}+\left|T^{t / 2} u_{0}\right|_{H}\right),
$$

and if $u_{0}$ belongs to $H^{2}\left(\mathbb{R}^{N}\right)^{m}$

$$
\left|W^{t} u_{0}-T^{t} u_{0}\right|_{H} \leq C\left(u_{0}\right) t^{2}\left(\left|u_{0}\right|_{H^{2}}+\left|T^{t / 2} u_{0}\right|_{H^{2}}\right) .
$$

Proof. We only prove (4.23). We notice that

$$
\begin{aligned}
\left|W^{t} u_{0}-T^{t} u_{0}\right|_{H} \leq & \frac{4}{3}\left|Z^{t / 2} Z^{t / 2} u_{0}-Z^{t / 2} T^{t / 2} u_{0}\right|_{H} \\
& +\frac{4}{3}\left|Z^{t / 2} T^{t / 2} u_{0}-T^{t} u_{0}\right|_{H} \\
& +\frac{1}{3}\left|T^{t} u_{0}-Z^{t} u_{0}\right|_{H} .
\end{aligned}
$$

We can estimate (4.26) thanks to (4.6). We also observe that we infer from (1.10) and (4.2) that

$$
\left|Z^{t / 2} Z^{t / 2} u_{0}-Z^{t / 2} T^{t / 2} u_{0}\right|_{H} \leq e^{\gamma t}\left|Z^{t / 2} u_{0}-T^{t / 2} u_{0}\right|_{H} \leq C\left(u_{0}\right) t^{2}\left|u_{0}\right|_{H^{2}} .
$$


We now have to work with the last term (4.25). We already notice that $T^{t} u_{0}=$ $T^{t / 2} T^{t / 2} u_{0}$, thus we infer from (4.6) that

$$
\left|Z^{t / 2} T^{t / 2} u_{0}-T^{t} u_{0}\right|_{H} \leq C\left(u_{0}\right) t^{2}\left|T^{t / 2} u_{0}\right|_{H^{2}},
$$

so our claim is proved.

4.3. Convergence result. We will use the following result.

Lemma 4.10. If $F$ satisfies (4.1), there exists a constant $C_{0}>0$ depending only on $\gamma$, such that for all $u_{0}$ and $v_{0}$ in $H$ and all $t \in[0,1]$,

$$
\left|W^{t} u_{0}-W^{t} v_{0}\right|_{H} \leq\left(1+C_{0} t\right)\left|u_{0}-v_{0}\right|_{H} .
$$

Proof. We rewrite $W^{t} u_{0}-W^{t} v_{0}$ as

$$
\begin{aligned}
W^{t} u_{0}-W^{t} v_{0}= & Z^{t / 2} Z^{t / 2} u_{0}-Z^{t / 2} Z^{t / 2} v_{0} \\
& +\frac{1}{3}\left(Z^{t / 2} Z^{t / 2} u_{0}-Z^{t / 2} Z^{t / 2} v_{0}-Z^{t} u_{0}+Z^{t} v_{0}\right) .
\end{aligned}
$$

We deduce from (1.10), (4.2) that

$$
\left|Z^{t / 2} Z^{t / 2} u_{0}-Z^{t / 2} Z^{t / 2} v_{0}\right|_{H} \leq e^{\gamma t}\left|u_{0}-v_{0}\right|_{H} .
$$

Since for $t \in[0,1]$, there exists $C_{1}>0$ depending only on $\gamma$, such that $e^{\gamma t} \leq 1+C_{1} t$, we can see that

$$
\left|Z^{t / 2} Z^{t / 2} u_{0}-Z^{t / 2} Z^{t / 2} v_{0}\right|_{H} \leq\left(1+C_{1} t\right)\left|u_{0}-v_{0}\right|_{H} .
$$

Denote $w=Z^{t / 2} Z^{t / 2} u_{0}-Z^{t / 2} Z^{t / 2} v_{0}-Z^{t} u_{0}+Z^{t} v_{0}$. Our purpose is now to prove that there exists a constant $C>0$ such that $|w|_{H} \leq C t\left|u_{0}-v_{0}\right|_{H}$. We let

$$
w_{1}=Z^{t / 2} Z^{t / 2} u_{0}-Z^{t / 2} Z^{t / 2} v_{0} \text { and } w_{2}=Z^{t} u_{0}-Z^{t} v_{0} .
$$

We now use that $Y^{t} u_{0}$ verifies

$$
Y^{t} u_{0}=u_{0}-\int_{0}^{t} F\left(Y^{s} u_{0}\right) d s
$$

Thus

$w_{2}=X^{t} u_{0}-X^{t} v_{0}-X^{t / 2} \int_{0}^{t} F\left(Y^{s}\left(X^{t / 2} u_{0}\right)\right) d s+X^{t / 2} \int_{0}^{t} F\left(Y^{s}\left(X^{t / 2} v_{0}\right)\right) d s$

and

$$
\begin{aligned}
w_{1}= & X^{t} u_{0}-X^{t} v_{0} \\
& -X^{3 t / 4} \int_{0}^{t / 2}\left(F\left(Y^{s}\left(X^{t / 4} Z^{t / 2} u_{0}\right)\right)-F\left(Y^{s}\left(X^{t / 4} Z^{t / 2} v_{0}\right)\right)\right) d s \\
& -X^{t / 4} \int_{0}^{t / 2}\left(F\left(Y^{s}\left(X^{t / 4} Z^{t / 2} u_{0}\right)\right)-F\left(Y^{s}\left(X^{t / 4} Z^{t / 2} v_{0}\right)\right)\right) d s .
\end{aligned}
$$


We deduce that

$$
\begin{aligned}
w= & X^{3 t / 4} \int_{0}^{t / 2}\left(F\left(Y^{s}\left(X^{t / 4} Z^{t / 2} u_{0}\right)\right)-F\left(Y^{s}\left(X^{t / 4} Z^{t / 2} v_{0}\right)\right)\right) d s \\
& +X^{t / 4} \int_{0}^{t / 2}\left(F\left(Y^{s}\left(X^{t / 4} Z^{t / 2} u_{0}\right)\right)-F\left(Y^{s}\left(X^{t / 4} Z^{t / 2} v_{0}\right)\right)\right) d s \\
& -X^{t / 2} \int_{0}^{t}\left(F\left(Y^{s}\left(X^{t / 2} u_{0}\right)\right)-F\left(Y^{s}\left(X^{t / 2} v_{0}\right)\right)\right) d s,
\end{aligned}
$$

and we infer from (1.10), (4.2) and the boudedness of $F$ that $|w|_{H} \leq C t\left|u_{0}-v_{0}\right|_{H}$, where $C$ depends only on $\gamma$. This concludes the proof of Lemma4.10.

Theorem 4.11. For all $u_{0}$ in $\mathbf{L}_{1}$ and for all $\tau>0$, there exists $C$ and $h_{0}$ such that for all $h \in\left(0, h_{0}\right]$, for all $n$ such that $n h \leq \tau$

$$
\left|\left(W^{h}\right)^{n} u_{0}-T^{n h} u_{0}\right|_{H} \leq C h|\ln h|\left|u_{0}\right|_{H} .
$$

Proof. We fix $\tau>0$ and we reduce the proof to the case where $F$ has a compact support and satisfies (4.1). The triangle inequality gives

$$
\left|\left(W^{h}\right)^{n} u_{0}-T^{n h} u_{0}\right|_{H} \leq \sum_{j=0}^{n-1}\left|\left(W^{h}\right)^{n-j-1} W^{h} T^{j h} u_{0}-\left(W^{h}\right)^{n-j-1} T^{(j+1) h} u_{0}\right|_{H},
$$

and we infer from Lemma 4.10 that

$$
\left|\left(W^{h}\right)^{n} u_{0}-T^{n h} u_{0}\right|_{H} \leq \sum_{j=0}^{n-1}\left(1+C_{0} h\right)^{n-j-1}\left|W^{h} T^{j h} u_{0}-T^{h} T^{j h} u_{0}\right|_{H} .
$$

For the case $j=0$, it follows from (4.22) that

$$
\left|W^{h} u_{0}-T^{h} u_{0}\right|_{H} \leq C\left(u_{0}\right) h\left(\left|u_{0}\right|_{H}+\left|T^{h / 2} u_{0}\right|_{H}\right) .
$$

For $j \geq 1$, we notice that $T^{j h} u_{0}$ belongs to $H^{2}\left(\mathbb{R}^{N}\right)^{m}$ and we use (4.23) to obtain

$$
\left|W^{h} T^{j h} u_{0}-T^{h} T^{j h} u_{0}\right|_{H} \leq C\left(T^{j h} u_{0}\right) h^{2}\left(\left|T^{j h} u_{0}\right|_{H^{2}}+\left|T^{(j+1) h} u_{0}\right|_{H^{2}}\right) .
$$

But for all $j h \leq \tau, C\left(T^{j h} u_{0}\right)$ is bounded, and we deduce from Lemma 4.4 that

$$
\left|T^{j h} u_{0}\right|_{H^{2}}+\left|T^{(j+1) h} u_{0}\right|_{H^{2}} \leq \frac{C}{j h} e^{C j h}\left|u_{0}\right|_{H} .
$$

Thus we obtain with 4.29)

$$
\left|\left(W^{h}\right)^{n} u_{0}-T^{n h} u_{0}\right|_{H} \leq C\left(u_{0}\right)\left(\sum_{j=1}^{n-1} \frac{e^{C_{0}(n-j-1) h} h}{j}+h\right)\left|u_{0}\right|_{H} .
$$

For small $h$, we have

$$
h \sum_{j=1}^{n-1} \frac{e^{C_{0}(n-j-1) h}}{j} \leq e^{C_{0} \tau} h|\ln h|
$$

and we obtain

$$
\left|\left(W^{h}\right)^{n} u_{0}-T^{n h} u_{0}\right|_{H} \leq C h|\ln h|\left|u_{0}\right|_{H} .
$$

This concludes the proof of Theorem 4.11 
Remark 4.12. The previous estimate is better than the one given in [2], with a rate of $h|\ln h|$ instead of $\sqrt{h}$ but unfortunately far from the fourth order. One way to increase the rate of convergence is to assume that $u_{0}$ is smoother, for example if $u_{0}$ belongs to $H^{2}$ we do not need to use the regularizing effect of $T^{t}$ and we obtain an estimate with a rate of $h$. We can also see that a similar proof of Theorem 3.10 can show that

$$
\forall t \in[0,1], \quad\left|e^{t M \Delta} e^{-2 t V} e^{t M \Delta}-e^{2 t(M \Delta-V)}\right|_{\mathcal{L}\left(H^{4}, L^{2}\right)} \leq C(c) t^{3}
$$

and in that case the rate is of $h^{2}$. Unfortunately, for the scheme $W^{t}$, we cannot do better (i.e., an order greater than 2) because of estimates (4.24), (4.25), (4.26) and working directly with $W^{t}$ seems to be very hard.

\section{ACKNOWLEDGMENTS}

I would like to thank Professor M. Schatzman for suggesting this work and for providing helpful comments.

\section{REFERENCES}

[1] S. Descombes, M. Schatzman, Directions alternées d'ordre élevé en réaction-diffusion, C. R. Acad. Sci. Paris Sér. I Math. 321 (1995), no. 11, 1521-1524. MR 96g:65104

[2] S. Descombes, M. Schatzman, On Richardson Extrapolation of Strang's Formula for Reaction-Diffusion Equations, in Equations aux Dérivées Partielles et Applications, articles dédiés à Jacques-Louis Lions, Gauthier-Villars Elsevier, Paris, 1998. MR 99i:65006

[3] B. O. Dia, M. Schatzman, Commutateurs de certains semi-groupes holomorphes et applications aux directions alternées, RAIRO Modèl. Math. Anal. Numér. 30 (1996), no. 3, 343-383. MR 97e:47055

[4] B. O. Dia, M. Schatzman, An estimate of the Kac transfer operator, J. Funct. Anal. 145 (1997), no. 1, 108-135. MR 98h:47057

[5] B. O. Dia, M. Schatzman, On the order of extrapolation of integration formula, Preprint no 275, Equipe d'Analyse Numérique Lyon-Saint Etienne, UMR 5585, 1998.

[6] P. de Mottoni, M. Schatzman, Geometrical evolution of developed interfaces, Trans. Amer. Math. Soc., Volume 347, Number 5, (1995), 1533-1589. MR 2000a:35022

[7] D. Goldman, L. Sirovich, A novel method for simulating the complex Ginzburg-Landau equation, Quart. Appl. Math. 53 (1995), no. 2, 315-333. MR 96a:35190

[8] D. Goldman, T. Kaper, Nth-order operator splitting schemes and nonreversible systems. SIAM J. Numer. Anal. 33 (1996), no. 1, 349-367. MR 97a:65063

[9] B. Helffer, Around the Transfer Operator and the Trotter-Kato Formula, Operator Theory: Advances and Appl. 78, 161-174 (1995). MR 96k:82013

[10] D. Henry, Geometric theory of semilinear parabolic equations. Lecture Notes in Mathematics, 840. Springer-Verlag, Berlin-New York, 1981. MR 83j:35084

[11] T. Ichinose, S. Takanobu, Estimate of the difference between the Kac operator and the Schrödinger semigroup, Comm. in Math. Phys. 186, (1997) 167-197. MR 99e:47052

[12] Q. Sheng, Solving linear partial differential equations by exponential splitting, IMA J. Numer. Anal. 9, Number 2, (1989), 199-212. MR 90g:65163

[13] J. Smoller, Shock waves and reaction-diffusion equations. Springer-Verlag, New York, 1994. MR 95g:35002

[14] G. Strang, Accurate Partial Difference Method. I: Linear Cauchy Problems, Arch. Rat. Mech. Anal. 12, (1963), 392-402. MR 26:4489

[15] G. Strang, On the Construction and Comparison of Difference Schemes, SIAM J. Numer. Anal. 5, (1968), 507-517. MR 38:4057

Unité de Mathématiques Pures et Appliquées, CNRS UmR 5669, Ecole Normale Supérieure de Lyon, 46, Allée d'Italie, 69364 lyon Cedex 07, France

E-mail address: stephane.descombes@umpa.ens-lyon.fr 